documentation was developed, staff engagement activities were carried out and data was collected.

Patient feedback was obtained and Goal Attainment Scores (GAS) used to measure achievement of personal goals.

Results Our mid-point review demonstrated positive changes, including adaptions in the delivery of care to focus more on patient-centred rehabilitative goals across the multidisciplinary team. Data collection is continuing and the final evaluation will take place in July 2018.

Conclusion Throughout the implementation of the project, challenges were identified in terms of the difficulty of initiating and maintaining the momentum of a cultural shift which has been previously evidenced. The final evaluation will therefore take account of both the objective outcomes and our reflections on how these challenges were addressed.

\section{P-135 DECONDITIONING, THE HIDDEN MENACE, IN THE PALLIATIVE CARE PATIENT}

Amanda Campbell, Karen Kidsley, Alison Hooper, Marie Magee. St Margaret's Hospice, Taunton, UK

\subsection{6/bmjspcare-2018-hospiceabs. 160}

Background Deconditioning can masquerade as general fatigue and disease progression, impacting function, confidence, reducing independence and quality of life, increasing the need for adaptions and care. It is a hidden menace. Deconditioning can be reversed with achievable focused strengthening exercises; this is good news for the palliative care patient.

Aims To enable identification and management of deconditioning in palliative care patients. To improve outcomes for deconditioned palliative care patients.

Method Create a screening process and exercise programmes for healthcare professionals to identify and advise individuals. Provide internal and external training for professionals. Provide online resources for professionals and patients. Provide weekly 'pop-up gym' sessions within the hospice for patients to receive individually prescribed exercise programmes. Provide regular talks for patients within the Sunflower Centre, highlighting the importance of exercise and the risks associated with deconditioning.

Results Deconditioning screening tools, each with decisionmaking guide and subsequent exercise programme created (2015). Deconditioning training workshops for professionals (2015), extended to external providers (2017). Word documents created (2015), with online resources of video clips (2016), supporting patients and professionals. Patient resources both generic and individually prescribed according to need. Patients have regained independence within short weeks using the advice and exercise. Healthcare professionals have replicated these results following the training. Positive patient feedback has been received:

'the greatest benefits are it has given me the strength and belief in myself'

'feeling strong enough to pursue further treatment options'

'Improved my quality of life... be more positive... enjoy life as much as possible.'

Gym sessions and talks began (2018) within the Sunflower Centre. Nine patients participated with personalised outcome measures identifying the programme benefits.

Conclusion Results indicate positive qualitative feedback and increased professional awareness. Progression requires quantitative data, increased signposting for recognition and prevention, along with professional practice support in identification and advice.

\section{P-136 ENABLING QUALITY OF LIFE BY A REHABILITATIVE APPROACH OF ADDING LIFE TO DAYS}

Lisa Wright. Kirkwood Hospice, Huddersfield, UK

\subsection{6/bmjspcare-2018-hospiceabs. 161}

Utilising a grant awarded by St James's Place Charitable Foundation we have created a culture of rehabilitation, enabling true patient-centred care in order to work towards the benchmark for best practice as set out in the 'How rehabilitative is your hospice' guidance by Hospice UK. A hospice wide approach has been adopted in order to facilitate and establish a culture shift across support and therapy day services and the hospice's in-patient unit with wider effects tangible across the organisation. A wide range of disease specific self-management groups have been created with full MDT involvement, goal setting has been a key focus and patient passports to assist with self-management of symptoms have been created.

Goal setting in order to achieve a rehabilitative and enabling approach has been the main focus within the inpatient unit and has required a culture shift away from disabling patients with our approach to care and having a much more individualised goal-focused approach to the whole multidisciplinary team delivery of care.

Outcomes have been measured by observing changes in Patient Outcome Scores and sometimes Karnofsky scores together with successful achievement of individual goals and feedback from patients.

This approach has supported the 292 attendance within the in-patient unit from 1 April 2017 to 31 March 2018. A review of the training sessions undertaken across all internal staff members, trustees and volunteers has identified approximately 150 indirect beneficiaries.

The creation of a team of enabling volunteers has helped to discover what really matters to patients across all hospice services. By using one-page profiles we are establishing personal activities, goals and areas that patients wish to participate in or talk over. These activities have relevance and meaning giving them a sense of purpose to exist and enabling them to live until they die.

\section{P-137 HOW A NURSING TASK FORCE HAS FOCUSED ON REMODELLING PRESSURE ULCER MANAGEMENT ON A HOSPICE IN-PATIENT UNIT}

Rose Davis, Rebekah Ashley, Jo Carby, Natalie Ward, Yvonne Tague. Wigan and Leigh Hospice, Wigan, UK

10.1136/bmjspcare-2018-hospiceabs. 162

\section{Drivers for change}

- The investigative process into a significant event highlighted inaccuracies in the nursing documentation

- Data capture for clinical governance meetings were inconsistent

- NICE pressure ulcer guidelines not fully implemented. 
Aim To improve the nursing management and prevention of pressure ulcers on the in-patient unit (IPU) in accordance with best practice and evidence. To debunk the myth that pressure ulcers are unavoidable at end of life.

Method Initial audit (Hospice UK) in 2016 highlighted a required review of the hospice policy and nursing documentation on admission and discharge. A task group commenced May 2016 comprising of the clinical director, IPU manager, $\mathrm{H} @ \mathrm{H}$ manager and IPU nurses with expertise and special interests in tissue viability.

\section{Action plan}

- Develop and adopt a policy and guidelines for the prevention and management of pressure ulcers on the IPU

- Collaborative working with the local NHS Trust to ensure we are working cohesively and consistently

- To provide training for IPU staff to develop their knowledge of pressure ulcers, risk assessments, management and prevention

- Raise awareness of the importance of skin care with patients, families and carers

- Improve the nursing documentation and reporting procedures, data collection and root cause analysis of pressure ulcers.

Results Audit results

\begin{tabular}{lc}
\hline Date & Average compliance $\%$ \\
\hline August 2016 & $34 \%$ \\
September 2018 & $92 \%$ \\
\hline
\end{tabular}

Pressure ulcers 2017

\begin{tabular}{lc}
\hline $\begin{array}{l}\text { Pressure Ulcer } \\
\text { Grade }\end{array}$ & Number \\
\hline Grade 2 & 3 \\
Grade 3 & 1 \\
Grade 4 & 0 \\
\hline
\end{tabular}

Pressure ulcers 2016

\begin{tabular}{lc}
\hline Pressure Ulcer Grade & Number \\
\hline Not known & 29 (unreliable data) \\
\hline
\end{tabular}

Conclusion Pressure ulcers are one of the most common occurring harms in healthcare. This task force has been the catalyst for the IPU nurses to challenge the myth of inevitable skin damage at end of life, underlining the importance of general nursing care interventions within a specialist palliative care unit.

\section{P-138 I AM A GUEST IN YOUR ORGANISATION}

Christina Ginsburg. Mountbatten, Isle of Wight, UK

10.1136/bmjspcare-2018-hospiceabs. 163

Background The discharge of a patient from hospital, who is nearing the end of life requires skilled, careful coordination and communication and can be highly time consuming. The Hospital Palliative Care Discharge Facilitator role is responsible for actively supporting discharge to people's preferred place of care. Research suggests that responsive, seamless discharges most often occur when one person is dedicated to the function to ensure that all aspects of the discharge process have been covered and nothing has been missed. The post holder was outreached from the hospice to the district general hospital for one year.

Aims Reduction in hospital beds days for patients at the end of life who do not wish to die in hospital. Prevention of hospital admissions by ascertaining patient's wishes, completing their advance care plan in the Emergency Department. Free NHS staff from organising care packages.

The objectives of the role:

- To identify daily patients who are end of life

- Expedite complex discharges by attending all wards and the Emergency Department

- Offer advice, support and consider available options with discharge plans.

Results

- 108 successful discharges in last nine months to own home or care setting

- 58 people were not discharged often due to a late referral and died during discharge planning or packages of care were unsourced.

Conclusion One of the values of the hospice is that we are innovative and bold - this is played out daily across the hospital. The role is responsive and creative. The success of coordinating complex discharges in a busy acute setting is supported by the hospice philosophy. There is a refreshing and unique quality about practising in an organisation you are not accountable to. The ability to challenge and question is well received and respected.

\section{P-139 I EXPERIENCE VR' - LOROS HOSPICE DEVELOPMENT OF VIRTUAL REALITY THERAPEUTIC DISTRACTION THERAPY APP}

Elaine Godber. LOROS Hospice, Leicester, Leicestershire

\subsection{6/bmjspcare-2018-hospiceabs. 164}

Background One year on from commissioning the first virtual reality (VR) film of a local park aimed at bringing relaxation and distraction therapy to patients whose lives have become restricted due to their illness the chance to see the world from the comfort of their chair or bed, LOROS Hospice has developed the project into a growing library of therapeutic films stored safely within its 'I Experience VR' App.

A robust evaluation study of patient response is underway to establish the impact on transforming patient care. To reach out to more patients it has engaged with other hospices and care homes with an invitation to join a partnership consortium and is constantly re-evaluating its offering.

Aim Provide patients with relaxation, calmness and provoke heart-warming conversations, a patient feels like they are actually there. It is simple to use for all health care professionals offering a different way to engage with patients and their families. 'I Experience VR' is subscription based with funds put directly back into the production of further 360 degree immersive VR films chosen by patients, residents and partners. 